\title{
USING A CATEGORY TO ACCOMPLISH RESISTANCE IN THE CONTEXT OF AN EMERGENCY CALL: MICHAEL JACKSON'S DOCTOR
}

\author{
Israel Berger, Celia Kitzinger and Sonja J. Ellis
}

\begin{abstract}
We report a single case analysis of a recorded emergency call with particular reference to the use of the non-recognitional categorical person reference 'a personal doctor' in the sequential context created by the Medical Priority Dispatch System (MPDS) protocol routinely used by the emergency services. We describe both the position and the composition of the turn in which this categorical person reference is deployed in order to analyse the action accomplished by its selection. We show how this category reference is selected to support the action in which the speaker is otherwise engaged, which is to resist the sequential trajectory proposed by his interlocutor (giving instructions for cardiopulmonary resuscitation). Our analysis makes two key contributions: 1) it provides a concrete detailed exemplar of how analysts can ground claims about category-bound inferences in the empirical practices of talk in interaction and 2) it extends existing work on emergency calls by relating their sequential structure to the MPDS protocol.
\end{abstract}

Keywords: Conversation Analysis; Membership Categorization Analysis; Person reference; Emergency calls; Resistance; Healthcare.

\section{Introduction}

The single-case analysis reported here analyses the use of a categorical person reference in the sequential context of an emergency call. It is distinctive in (a) exposing the use of a categorical person reference to accomplish 'resistance' to the trajectory of action proposed by another speaker and (b) relating the sequential unfolding of an emergency call to the Medical Priority Dispatch System (MPDS) protocol which is supposed to underpin it.

There is a long history of research on how speakers use categories ('baby', 'mother', 'doctor' etc.) in talk-in-interaction, beginning with the ground-breaking work of Harvey Sacks (1995) and the two distinct research traditions that emerged from his approach: Conversation Analysis which focuses on the sequential organisation of talk (e.g. Schegloff 2007), and Membership Categorization Analysis (e.g. Hester \& Eglin 1997). Despite some recent attempts to unite them (e.g. Stokoe 2012), these two branches of Sacks's work have 'developed largely in isolation from one another' 
(Plunket 2009: 24) and, as Stokoe (2013: 279) points out, 'categories and sequences have rarely been studied concurrently'.

Equally, there is a long history of conversation analytic research on calls to the emergency services, but curiously none of it engages with the Medical Priority Dispatch System (MPDS, http://www.prioritydispatch.net). The MPDS, originally invented by Dr. Jeff Clawson in the late 1970s and subsequently developed, updated and constantly refined by the National Academy of Emergency Medical Dispatchers, is generally accepted as the international standard for emergency medical dispatch care and practice (Clawson \& Dernocoeur 2003). Most conversation analytic work relies on the five-part structure proposed by Zimmerman (1984) which proposes that emergency calls consist of: Opening, request, interrogative series, response and closing. The MPDS protocol in which emergency dispatchers are trained advocates that dispatchers begin with a call receipt (broadly, Zimmerman's 'opening'); it omits any reference to a 'request' (perhaps because it is focused on what dispatchers should do rather than on what callers might say) and moves straight to 'Case entry interrogation' and the 'Four Commandment' questions which define what Zimmerman refers to as an 'interrogative series'. As prescribed by the protocol, the questions in this series are organised by reference to a algorithmic flow-chart and designed to arrive at crucial diagnostic information as rapidly and efficiently as possible. Although conversation analysis of emergency calls frequently describe an 'interrogative series', none of them relate this to the series of questions proposed in the MPDS in which the dispatchers whose talk is being analysed have been trained (see for example Whalen \& Zimmerman 1990; Chase 2014; Garcia 2015). Meanwhile, a parallel stream of research evaluates the MPDS system, correlating protocol compliance/adaptations with outcome measures (e.g. Heward 2004; Cheung et al. 2007) but without using conversation analysis. Our analysis uses the protocol, and observed departure from it, as a resource for inspecting the sequential unfolding of an emergency call. This MPDS protocol provides the context within which the use of a category to divert a sequential trajectory can be understood.

Researchers who are interested in how categories are selected and used in talkin-interaction have observed that they are not merely taxonomic labels but are deployed to support or constitute the actions speakers mean to be doing with their turns (Schegloff 2007a: 471). In selecting categories for reference, a speaker is 'fitting the precise referring expression to the type of pragmatic action that they are otherwise performing' (Stivers 2007: 73). A key issue is '[h]ow ... the ordinary workings of talk or other conduct in interaction serve to get categorization devices made relevant or activated for the parties' (Schegloff 2007a: 477). This paper offers a conversation analytic case study of a categorical person reference as it is deployed in a single interaction. Single-case analyses can be particularly useful for explicating analytical nuances that are uncommon or may be obscured by larger scale studies (cf. Schegloff 1987; Toerien \& Kitzinger 2007; Whalen et al. 1988). We will show how a categorical person reference is used to resist the course of action that is being pursued by an interlocutor and how the inferences it makes relevant influence the ongoing interaction.

It is common for researchers to assert (following Sacks 1972a, b) that categories are consequential in talk, since they lead to inferences about the people so categorised and the kinds of activities in which they might engage (i.e. categories are 'inferencerich' and associated with 'category-bound activities'). This means that categories store 
'a great deal of the knowledge that members of a society have about the society' (Sacks 1995: 40-41). As analysts, our task is to 'discover' what it is that members know, and not to impose what we already know (as co-cultural members) upon the data. For example, as cultural members, we know that someone categorised as 'a doctor' is, by virtue of that category membership, presumed competent to perform cardiopulmonary resuscitation (CPR), but that no such competence is associated with someone categorised as 'a Freemason' or 'a bankrupt'. However, Schegloff (2007a) cautions against using our vernacular knowledge as cultural members to warrant such claims, 'There must be analysis to show the claim is grounded in the conduct of the parties, not in the beliefs of the writer' (Schegloff 2007a: 476). That is the approach exemplified here; our analysis explores the relationship between an articulated category (such as doctor) and the actions of the turn, the sequence, and the overall trajectory of the interaction.

The present analysis draws on and contributes to the conversation analytic literature on person reference (Lerner \& Kitzinger 2007; Sacks \& Schegloff 1979; Schegloff 1996) and membership categorization (Kitzinger 2005; Kitzinger \& Rickford 2007; Sacks 1972a, b; Schegloff 2007a; Stokoe 2009; Wilkinson \& Kitzinger 2003). In particular, we aim to build on this work by showing how analysts' understanding of the inferences associated with a category can be grounded in speakers' practices.

\section{The data}

We focus on a categorical person reference used in the course of a nearly two-minute emergency call made to the Los Angeles Fire Department at 12:21pm on June 25, 2009. The call was released to major news companies and is widely available online (e.g., http://news.bbc.co.uk/1/hi/8121884.stm). We recommend that readers listen to the call in advance of, and in conjunction with, reading this article. A complete Jeffersonian transcript is provided in the Appendix. Extracts are numbered as they appear in the text, with line numbers corresponding to the full transcript. In addition, in order to better understand the interaction in our target 911 call (and given the absence of any previous conversation analytic research on the MPDS), we have found it useful to make comparisons with other recent (especially Californian) publicly available 911 calls which provide evidence about how the MPDS is usually oriented to by the dispatcher (and hence provide a means of warranting our observation of a departure from it in our target call). Extracts are provided and the data described as they become relevant.

The two speakers in our target call are the caller (Clr) and an emergency medical dispatcher (EMD) at a 911 public safety answering point, trained in the MPDS in accordance with the California Emergency Medical Services dispatch program guidelines (Watson et al. 2003) Although he does not self-identify by name on the audiotape, it is now known (and the Caller would himself have known at the time) that he was Mr. Alberto Alvarez, a security guard for Michael Jackson, the 'King of Pop' and one of the most commercially successful popular music artists of all time. He is making the call on behalf of Michael Jackson - referred to as 'a gentleman here that needs help'. It is also apparent from the call that there are at least two people present (in addition to Michael Jackson, who is not conscious). On the EMD's side, there is another EMD who makes a brief intervention. On the caller's side, there is 'a personal doctor here' who is briefly audible in the background. That doctor was known to the Caller as 
Dr. Conrad Murray, Jackson's personal physician. At the time of the call he was administering CPR.

Some additional ethnographic context is useful in understanding the context of the call and what the Caller would have known at the time of making the call. In his subsequent court testimony he said that he had been summoned to the room by Dr. Conrad Murray and that Michael Jackson appeared to him to be already dead at that point. He said he asked the doctor what had happened and was told that Jackson had a 'bad reaction'. The doctor then instructed him to bag up medical items - vials and a saline bag from an IV stand - before calling the emergency services about 25 minutes later (The Telegraph 2011). The post-mortem examination revealed that the medications propofol and lorazepam were the primary substances responsible for Jackson's death, (Moore 2009) and Conrad was convicted of involuntary manslaughter and sentenced to four years in prison (Deutsch 2011).

\section{Analysis}

The target of our analysis is the categorical person reference a personal doctor (line 52) produced just over one minute into the conversation. Person reference and membership categorization are 'quite distinct activities' (Schegloff 2007b: 456), but here referring to a person is accomplished using a category of which the referent is a member ('a personal doctor'). Our analysis describes (1) the position and (2) the composition of the turn in which the categorical person reference is deployed and then (3) focuses on the way in which the category is analysably selected in order to mobilize particular inferences in the service of the action in which the speaker is engaged.

\subsection{Position}

The position of our target turn can be described in two ways: (1) in relation to the overall structural organisation of the call as a whole and (2) in its immediate sequential context.

\subsubsection{Overall structural organisation}

This call conforms very closely to the MPDS protocol. It opens with an EMD response to the call (extract 1), which covers the first two 'elements' or phases of MPDS protocol-driven questioning:

(1) (Call to 911 Emergency Service made on 25 June 2009)

01 EMD: $\quad$ Fire ' $n$ paramedics thirty three what is the address

02 of your emergency:

Phase 1 is Call receipt: the identification of the service the caller has reached (as at line Fire ' $n$ paramedics thirty three (line 1). Phase 2 Case entry interrogation, i.e. a request for address and phone number, and this is launched with 'What is the address of your 
emergency', lines 1-2). The second phase continues (extract 2) with the Caller's response to the question about his address (lines 6-8), a confirmation check (lines 9-10), and a question and answer about the Caller's number.

(2) (Call to 911 Emergency Service made on 25 June 2009)

03 Clr: $\quad$ Yes Sir. I need to I- I- I need an ambulance

04 as soon as po:ssible sir.

05 EMD: 'kay Sir.=What's your a:ddress.

06 Clr: $\quad$ It's one hundred (.) North Carolwood Drive .hhh

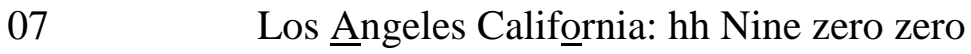

08 seven seven.

09 EMD: $\quad$ Did y'say Carolwoodi

$10 \mathrm{Clr}: \quad$ Carolwood Drive yes: hh. $\mathrm{hh}$

$11 \quad(1.5) /(($ Background conversation $))$

12 EMD: Okay Sir 'n what's the phone number you're

13 callin' from.

$14 \quad$ ((deleted material here) $)$

15 EMD: $\quad$ A:nd what's the problem.=Exactly what happened.

16

$(0.5)$

Phase 3 begins with line 15 and continues to line 33 - again in a 'textbook' fashion (extracts 3 \& 4). The MPDS literature refers to this phase as the Four commandments/questions (also called pre-entry primary survey, Clawson 2000). It consists of elicitation of a description of the chief complaint/medical problem (lines 1521 ), the patient's age (lines 23-25), the status of consciousness and the status of breathing (lines 26-32).

(3) (Call to 911 Emergency Service made on 25 June 2009)

15 EMD: A:nd what's the problem.=Exactly what happened.

$16 \quad(0.5)$

17 Clr: Uh sir I have a: we have a: .hh a: a: gentleman

18 here that needs he:lp and he:'s not brea:thing

19 yet. .hhh he's not breathing and we need

20 tuh- we're p- tryin' tah pump him but he's not

$21 \quad(0.2)$ he['s not br ${ }^{\circ}$ eathin'] so: ${ }^{\circ}$

22 EMD: [ $\quad$ o k a : y. ]

23 EMD: Oka:y how old is 'e.

$24 \quad$ (.)

25 Clr: He's uh fifty years old sir.=

26 EMD: $\quad=$ Fifty: ¿ $\underline{\text { Oka:y. }}$.hhh (.) He's unconscious he's

27 not brea:thingi

28 (.)

29 Clr: $\quad$ Yes he's not breathing sir.

30 EMD: $\quad$ Okay and 'e's not conscious eithe: $r_{i}$

31 [(He's) not breathin']

32 Clr: [ N o h e's n ot ] conscious si[:r.]

33 EMD:

34

[O]ka:y.

( ) .hh (1.0)/(Get 'im on the floor) 
Phase 4 is Immediate dispatch (when necessary). In the current call, this happens offline (lines 33-34). High performance MPD centres have two dispatchers for each emergency call, one to engage with the caller and the other to dispatch emergency units (Ornato 2009). A second dispatcher is just audible off-line apparently relaying the address to the paramedics dispatched to the scene (not transcribed). In our call, however, there are no post-dispatch instructions (as recommended by the protocol), to inform the caller that the ambulance has been sent, or that CPR instructions will be given in anticipation of its arrival. Instead CPR instructions are initiated right away (at line 35), and information about what the instructions are for and about the imminent arrival of the ambulance are instead provided later as reassuring responses to concerns raised by the caller (see lines 45-47). With the exception noted above, the EMD's talk displays close compliance to the MPDS protocol, and these four elements occupy the first 50 seconds of the call (until line 33).

Phase 5, Pre-arrival instructions, is then launched in accordance with the MPDS protocol for patients who are not conscious and not breathing. The EMD (possibly prompted by another dispatcher just audible at line 34) then launches this next phase, which consists of standardised instruction in basic life support skills. These instructions included the Heimlich manoeuver (for choking), emergency childbirth instructions, and - as here - cardiopulmonary resuscitation (CPR) for cardiac arrest ${ }^{1}$ (Clawson 2000). Overall, neurologically-intact survival rate for out-of-hospital cardiac arrest in Los Angeles was $1.4 \%$, and $2.1 \%$ for patients who receive bystander CPR (Eckstein et al. 2005). Average time (in Seattle) from the start of the MPDS-driven call to the start of CPR instructions is 75 seconds (Berdowski 2009: 2096). Since delayed recognition of cardiac arrest is attributable in part to vague descriptions of breathing and agonal (shallow and/or gasping) breathing (Berdowski 2009: 2096), the prompt launch of CPR instructions here (at 51 seconds) is attributable to the clear and reiterated report that the patient is 'not breathing' (lines 18, 19, 21 and 29).

(4) (Call to 911 Emergency Service made on 25 June 2009)

34

( ) .hh (1.0)/(Get 'im on the floor)

1 These scripts are regularly reviewed, their efficacy monitored, and their wording changed accordingly. It has not been possible to identify the precise script used by this particular dispatcher, but we have access to several variants of it (e.g., on CPR instruction, Deakin et al. 2007; Roppolo et al. 2005). The 8-point version of the CPR script supplied in Deakin et al. (2006: 438) starts as follows:

1. Patient to phone

Listen carefully and I'll tell you how to help her/him.

Get her/him as close to the phone as possible.

Do not hang up. Do it now and tell me when it's done.

Where is she/he now?

2. Check airway.

Listen carefully.

(Not breathing) Lay her/him flat on her/his back on the floor (ground) and remove any pillows.

Kneel next to her/him and look in the mouth for food or vomit (sick). Is there anything in the mouth?

It continues through scripts for [3] Check breathing; [4] Start mouth-to-mouth; [5] Check breaths; [6] CPR landmarks; [7] Compressions and [8] Continue CPR. Note that neither mouth-to-mouth nor checking a pulse is included in protocols for CPR instruction by EMD personnel used at the time of the call (Roppolo et al. 2005: 203). 
35 EMD: $\quad$ Alright. $>$ D'you have 'im- What- Is 'e on the

36

37 Clr:

38 EMD:

39

40

41 Clr:

42 EMD:

43

44

45 Clr:

46 EMD:

47

48

49 Clr:

50 EMD: floo: $\mathrm{r}_{i}=$ Where is 'e at right no: $\mathrm{w}_{i}<$

He's on the bed sir. He's on the [be:d.]

get 'im on the floo:r,

[ Oka:]y let's

(0.5)

Okay?

Okay let's get 'im down to the floo:r.=I'm gunna

help you with C P R right no:w oka::yi

(.)

W::e need 'im to get- we need a: [(

[Yes: We're] already

on our way the:re. We're on our way. $=$ I'm

gunna do [what I ]can tuh help you over the pho:ne.

$\left[\left(\begin{array}{ll}) \\ \end{array}\right]\right.$

We're already on our wá:y.

The scripted CPR protocol requires the dispatcher first to enquire about where the patient is right now (as at lines 35-36) and then to instruct the caller to lay him flat on his back on the floor (lines 38-39). There is no provision for asking permission or gaining informed consent, both of which are now considered forms of dispatcher malpractice. With the sole exception of expected deaths with the confirmed presence of a Do Not Resuscitate order, dispatchers are trained to presume that the caller consents to carrying out life-saving attempts, and that the patient consents to receiving them (Clawson 1991). Nonetheless, it is very common for callers to refuse to follow emergency CPR instructions. One study found that across $168 \mathrm{CPR}$-instruction-relevant calls, only 25 people actually received CPR. Leading reasons why CPR was not administered were that callers explicitly refused to do CPR (18\%) and callers disconnected the phone before instructions were complete (19\%). The remainder were either emotionally or physically unable to comply (Lerner et al., 2008).

This caller, too, is resisting CPR instructions. The first evidence of resistance is his lack of response (line 40) and his delayed okay (line 41), which, although at face value a compliance marker, has upward intonation conveying some problem with understanding why this instruction is issued. The EMD apparently hears it this way, as he reissues the instruction (line 42) and provides an account for it (I'm gunna help you with CPR right now, lines 42-3) with a tag question designed to elicit acceptance (okay, line 43). Further resistance follows, however. First the silence (line 44) where the caller is not accepting the EMD's proposal of help with CPR, then an explicit alternative proposal - that the patient needs hospital treatment/an ambulance (although the last word is lost in overlapping talk, line 45). The EMD tackles the caller's objection by accepting the need for trained medical assistance (Yes, line 46) and reassuring the caller that help is on its way such that his own CPR efforts would be merely an interim measure (lines 46-48). He continues in the face of an incipient interruptive turn from the caller (line 49), recycles the reassurance that we're already on our way (line 50) and then continues to hold the floor with a big pre-beginning in-breath and a new turn constructional unit which initiates a new action.

Dispatchers are taught that if callers interrupt the EMD's pre-arrival instructions with questions or concerns about whether the ambulance is on its way, this is due to 
'gaps' (the term is not used in the conversation analytic sense here) in the EMD's interrogation and treatment talk that allow the caller to seize control of what should properly be a dispatcher-directed interaction (Clawson \& Dernocoeur 2003). 'Avoided gaps' is one of the assessment items on the Medical Dispatch Case Evaluation Record used in assessing EMD compliance with the protocol (Dispatch Magazine Online, retrieved January 30, 2010). In this call, the EMD avoids any such 'gaps' and seeks to re-establish control and avert further resistance from the caller. He holds on to his turn and momentarily sidesteps the CPR issue, instead asking about the onset of the patient's medical problem (line 50, see analysis below). It is in response to this attempt to regain control that the caller produces the categorical person reference.

One way of describing the position of our target person reference formulation, then, is that it arises in the context of the EMD's attempt to issue pre-arrival instructions for performing CPR to a resistant caller, i.e. one who has not indicated willingness to comply with these instructions and who has proposed (what he, the caller, has treated as) an alternative course of action.

\subsubsection{Immediate sequential context}

A second way of describing the position of the target person reference formulation is in its immediate sequential context. It is selected as part of the design of the second pair part of an adjacency pair (Schegloff 2007c: 13) in which the speaker is answering a question.

(5) (Call to 911 Emergency Service made on 25 June 2009)

50 EMD: $\quad[\ldots] . h h h h$ Did anybody see hi:mi

51

52 Clr: $\quad$ Ye:s uhm we have a personal: doctor here with him sir.

The EMD's question (at line 50, extract 5) is a poorly designed version of a key question in the cardiac/respiratory arrest protocol, did you see what happened. Some version of this question is standard across the contemporary 911 emergency calls we have accessed on the web. It is designed to establish whether the person who is not conscious and not breathing may have, for example, been electrocuted, choked on something, reported chest pain, or been hit on the head. Answers are relevant to the provision of subsequent pre-arrival instruction and may also be passed to responding paramedics and may influence, for example, the kind of equipment they initially take to the patient on arrival. The question is usually formatted as did anybody see what happened or - as subsequently recycled in the current call - did anybody witness what happened (line 66). Recipients usually display their understanding of this question as designed to elicit an account of what may have precipitated the patient's collapse by giving, in the next turn, an account of the patient's prior physical condition. For example, in another (Californian) 911 call made a few months after the call under analysis here, also on behalf of an adult who is not breathing, the recipient responds by reporting the patient's prior symptoms (lines 2-3, extract 6). 
(6) (Mother calling 911 on behalf of Brittany Murphy, 8:00 20 December 2009; http://www.youtube.com/watch?v=5pnHf0_b6-U)

01 EMD: Did anybody see what happened;

02 Clr: $\quad$.hhhh ((sobbing in-breath)) She was dizzy she

03

04 couldn't walk right she's got a co:ld .hhhh oh my go(h)d huh huh

Whereas the responsive turn in extract 6 cooperates with the diagnostic agenda of the question, the responsive turn in extract 7 does not. Instead of reporting on the patient's observable state prior to collapse, the caller reports the identity of the person who was in a position to make such observations. Notice that when this question-answer pair is recycled (lines 66-70), the EMD treats the answer (Just the doctor sir, line 68) as unsatisfactory (by waiting for a more adequate response, line 69) and follows up on The doctor's been the only one here (line 70) by pursuing a report of what the doctor might have seen (So'd the: doctor see what happened, line 71) - a question which the caller relays to the doctor (line 72) who also (line 73) apparently declines to provide any account for the patient's condition.

(7) (Call to 911 Emergency Service made on 25 June 2009)

66 EMD: Did anybody witness what happenedi

$67 \quad(0.5)$

68 Clr: $\quad$ Uh no. Just the doctor sir.

$69 \quad$ (.)

70 Clr: $\quad$ The doctor's been the only one here.

71 EMD: $\quad$ Okay. So'd the: doctor see what happened;

72 Clr: $\quad$ Uh uhm Doctor did you see what happened sir.

73 Doc: $\quad$ ((from distance)) ( ) co:me.

74 Clr: $\quad$ ( ) Sir you just- .hh uh uhm if you can plea:se

None of these three question-answer pairs (lines 50-52; lines 66-70; and lines 7173) about what might have been seen to have happened to cause the patient to stop breathing is answered with the sort of information which (on the basis of what has subsequently emerged about the conditions under which Michael Jackson went into cardiac arrest) it could have been, i.e. something like: 'He was given propofol about an hour and a half ago because he couldn't sleep - and he had diazepam, lorazepam and midazolam earlier today'. Neither the Caller (who has just arrived on the scene and may not have this information) nor the doctor to whom he subsequently relays the reissued question (lines 66 and 71) provides this information or anything like it. Thus a second way of describing the position of our target person reference formulation is in terms of its local sequential position as a second pair part that responds to the format of the question (who witnessed the collapse) but not to the action it is designed to implement (establishing what precipitants of the collapse that person might have seen) so as to assist resuscitation attempts. 


\subsection{Composition}

Conversation analysts have used the concept of 'resistance' to refer to turns at talk that 'either decline to fully address the topical or action agenda of the question, or shift to a different agenda, or both (Bull 1994; Bull \& Mayer 1993; Clayman \& Heritage 2002a; Ekstrom 2009a; Greatbatch 1986a; Harris 1991)' (Clayman 2013: 647). We have already described the Caller's response to the turns 'Let's get him on the floor' (lines 38-39) and 'I'm gunna help you with CPR now okay' as 'resistant' since both propose a course of action (preparing to initiate CPR and accepting instruction to do so) with which the Caller does not cooperate. For the EMD, the response to the turn 'Did anybody see him' is also one that fails to address the topical agenda of the question since we know from the MPDS protocol what this question is designed to elicit. Since the question is - on this occasion - poorly designed for the job it is intended to do, the 'resistance' may not be designedly obstructive in the way that the earlier responsive turns seem to be, but its effect nonetheless is clearly to block the action trajectory (initiating CPR) which the dispatcher is pursuing, and it does so more effectively than the previous 'resistant' responses (in that the dispatcher finally ceases his attempts to pursue CPR instruction).

Resistance has been explored by conversation analysts across a range of institutional contexts, including news interviews (Clayman 2013) and medical settings, e.g. resistance to professional advice (Heritage \& Sefi 1992), resistance to medical diagnosis (Gill 2010), and resistance to treatment recommendations (Koenig 2011). Resistance in emergency calls has been described in the form of rude, abusive, and hysterical behaviour (e.g. Clawson 1986; Tracy \& Tracy 1998; Whalen et al. 1988; Zimmerman 1992). By contrast, this caller displays resistance in talk that is hearably 'polite' and 'formal'. Compositional features of the turn (Yes uhm we have a personal doctor here with him sir, line 52) that contribute to this formality (and are consistent with the speaker's talk elsewhere in this conversation) are:

a) the formal address term sir (used repeatedly throughout this call), which displays a respectful stance toward the addressee. It is culturally appropriate for the caller to use formal terms of address and data extracts cited in the literature show the use of sir or ma'am from EMDs to callers (e.g. see Zimmerman 1992: 430, Extract 7 line 4; and 431, Extract 9, line 6). Although it can be expected that a security guard may refer to his employer or visitors as 'sir', this is an unusually polite address term from caller to EMD.

b) self-reference as we rather than $I$, which reflects the caller's predominant usage throughout this call, and brings off the effect of speaking formally as if as a representative of some organization rather than in an individual capacity (Compare the institutional use of we in other 911 calls quoted by Zimmerman 1992: We gotuh guy down here that's uh over intoxicated (a Greyhound bus depot security guard Zimmerman 1992: 454, Extract 55); We have an unconscious diabetic (bookstore representative, Zimmerman 1992: 455, Extract 57). He seems to be speaking as a representative of the household that employs him and not in any personal capacity. In line with this, he never self-identifies by name as some callers do (see data in Zimmerman 1992 and Clawson \& Dernocoeur 2003). 
c) reference to the person who may have witnessed the patient's collapse as $a$ personal doctor, which may be fitted to his earlier reference to the patient as $a$ gentleman ${ }^{2}$ since both are formal and 'distanced' ways of referring to persons, consistent with the caller's 'institutional' role and formal presentation. Other institutional representatives making 911 calls refer to the patient as a guy, an unconscious diabetic, a gal, or a customer (see data cited in Zimmerman 1992). These formulations contrast with other calls in which the patient is referred to as my wife, my husband, my brother, or my sister, and other people present are referred to as my son, my daughter, my sister (again, all in Zimmerman 1992), thereby evoking personal, familial relationships in contradistinction to the formal relationships evoked by a gentleman and a personal doctor. The indefinite article $a$ (where his personal doctor would have been possible) is another aspect of the 'formality' of this person reference.

d) Finally, the turn-initial yes claims that the speaker is answering with a preferred response, since the question is formatted grammatically to prefer a yes answer, and also prefers yes in terms of the action it is designed to implement (Schegloff 2007c: 58-63). Note that when the question is recycled (line 66), the recycled answer is instead no-prefaced. This revised answer acknowledges (as the initial answer in our target turn does not) that the question is designed to elicit a description of what happened. Whereas the turn-initial no admits that the answer will not advance the course of action the question has set in motion, the turninitial yes in our target line claims cooperation that is not then delivered - a claim to cooperation that contributes to the 'politeness' of the turn.

In sum, then, unlike resistant turns in other emergency calls (e.g., 'You don't know what the fuck you're even talking about', Tracy \& Tracy 1998: 246, Call 1, lines 4647 ), this caller's resistance is not visible/audible primarily through the composition of

2 One feature of this emergency call and others featuring celebrity patients is that although the possibility exists for use of a recognitional person reference, the celebrity is never referred to by name (i.e. the caller does not say we have Michael Jackson here... (lines 17-18) but we have a gentleman here...). In other 911 calls, for example, Tiger Woods (the famous golfer who drove his car into a tree) is referred to as a neighbor (I have a neighbor he hit the tree,

http://www.huffingtonpost.com/2009/11/29/tiger-woods-911-call-audi_n_372977.html);

Brittany Murphy (the actress who collapsed and died at her home) is initially referred to as somebody (somebody's passed out) and subsequently as my daughter (my daughter's passed out)

(http://www.youtube.com/watch?v=5pnHf0_b6-U); and Casey Johnson (heiress of Johnson \& Johnson) is also referred to as somebody (I have somebody who I believe has just died)

(http://www.huffingtonpost.com/2010/01/09/casey-johnson-911-call-re_n_417520.html).

Media commentators have noted that 'Even as Jackson lay dying, Alvarez would not break his ban on public use of his name, referring to him as "a gentleman" (Graham and Boffey 2009) and have used this observation - along with the fact that his neighbours said he had never revealed the name of his employer to them - as evidence that he was 'fiercely loyal'. However, recognitional reference forms (in particular, names) are not normally used when 911 calls are made on behalf of celebrities. This may be partly motivated by concerns about privacy or by anxiety that a claim to be calling on behalf of a celebrity may cast doubt on the authenticity of the call. More generally, though, withholding the unique identity of the patient (as opposed to medically-relevant features such as their age and gender) demonstrates understanding that it is not relevant to emergency medical treatment. We have located only one call in which a recognitional person reference form (the name of the patient, who is not a celebrity) is treated as relevant, and that is a call in which the EMD who takes the call is the mother of the young man who has been shot and whose voice is recognised by the caller (http://www.youtube.com/watch?v=YHT2nUhanyM). 
the turn, but rather through the action that its composition is designed to accomplish in its sequential context. ${ }^{3}$

\subsection{The categorical person reference}

We have seen so far that the categorical person reference a personal doctor is selected in designing a turn that is (as we have shown) analysably resistant (albeit in a polite/formal manner) both to the emergency medical dispatcher's proposed course of action (instruction in CPR) and - whether or not designedly so - to his immediately prior question. We now turn to the category label itself. As Schegloff (2007a: 469) has said, "Why actually should one care all that much about these terms and their deployment? What is the big deal about which 'category label' ... is applied to someone?'

We begin by reiterating - with specific application to this particular case - what other conversation analysts have already articulated. Given what we now know about the identity of the person to whom the caller is referring (Dr. Conrad Murray), it is apparent that there are a number of other descriptively accurate categorical terms that could - depending on the extent of the caller's knowledge at the time - have been used to do referring. These include (from a quick trawl of internet sources) 'an African American', 'a Freemason', 'a child support defaulter', 'a bankrupt', and 'a serial philanderer'. The question we ask as conversation analysts is: What is it that 'We have a personal doctor here with him sir' accomplishes in this interactional context.

As cultural members, we know that a doctor (rather than, say, a Freemason) is just the category of person to have around in a medical emergency, and so it would be easy to provide a vernacular gloss (in place of analysis) based on the kind of 'conceptual stipulation' that Schegloff (2007a: 479) critiques. Instead, we will now use the analyses we have developed thus far to arrive at an empirically grounded analysis that shows how this category is selected and deployed in this particular sequential context and how the inferences associated with it are displayed and oriented to by the interlocutors. We focus now on that part of the interaction (extract 8).

(8) (Call to 911 Emergency Service made on 25 June 2009)

50 EMD: $\quad[\ldots] . h h h h$ Did anybody see hi:mi

$51 \quad(0.2)$

$52 \mathrm{Clr}$ : Ye:s uhm we have a personal: doctor here with him sir.

$53 \quad$ [.hhh (But-)]

54 EMD: $\quad$ [Oh y'have a] doctor the:re;

55 (.)

$56 \mathrm{Clr}: \quad$ Yes. But he's not responding to anything. To no: .hh no:

$57 \quad$ He's not responding to: C P R or anythi[ng.]

58 EMD: [매:]:: Oka:y.

59 Well we're on our way the:re. If your guys're doing

3 Note that comparing turn designs across interactions is absolutely standard practice in conversation analytic research. At its most basic, it is how data collections are compiled and analysed. For a justification and explanation of the value of comparing talk in a target interaction with talk in other similar interactions, see Kitzinger 2008. 
Referring to the person who might have 'seen him' collapse using the categorical formulation 'a personal doctor' ${ }^{4}$ contributes to the ongoing resistance to initiating CPR (in a way that We have a Freemason here with him sir would not) by conveying, for the first time, the presence on the scene of a relevantly qualified expert whose presence might be taken to pre-empt the EMD's projectable next actions, since this expert might reasonably be assumed to have ensured that necessary preliminaries to CPR had been performed prior to engaging in CPR himself, rendering both actions unnecessary on the part of the EMD.

The EMD's response to this turn is hugely significant. He treats it as blocking the MPDS-governed trajectory upon which he had embarked and departs from his script. He stops (at least for now) pursuing the question of what happened to cause or precipitate the patient's medical problems, receipts the prior turn as news ( $O h$, line 54), and produces a modified repeat of it (line 54) by way of checking his understanding. Understanding checks are explicitly described as deviations - albeit permissible ones from the MPDS protocol:

Compliance to protocol shall mean the adherence to the written text or scripts and other processes within the approved medical dispatch protocol reference system except that, deviation from the text or script may only occur for the express purpose of clarifying the meaning or intent of a question or facilitating the clear understanding of a required action, instruction, or response from the caller. (Watson et al. 2003: 3)

After the caller confirms the EMD's understanding as correct (yes, line 56) and continues with action of trying to get an ambulance (perhaps defending against the possibility that already having a doctor already in attendance might be treated by the EMD as a reason not to send help, lines 56-57), the EMD provides an explicit account for abandoning his attempt to give CPR instruction. Unlike almost everything else the EMD says in the course of this call, his account for abandoning CPR is not scripted. There is no provision in the protocol for abandoning or for failing to attempt CPR on an unconscious patient who is not breathing except when that patient is very clearly already definitively dead (e.g., decapitated or decaying). (It is possible that this unscripted account is designed not only for his current interlocutor but also 'for the record', since EMDs are subject to a continuous quality improvement program that includes ongoing random case review of each EMD's calls for MPDS-compliance (Watson et al. 2003)). His account for discontinuing CPR instruction (and thereby

4 The description of this doctor as a personal doctor (as opposed simply to a doctor) may be designed to account for the doctor having been present in the period before the patient's collapse (and hence possibly in a position to see what happened to occasion it) rather than only having been called subsequent to the collapse. It also conveys (though perhaps without having been designed to do so) both that the doctor is someone who already knows the patient and his medical history. Note, however, that in his understanding check (line 54) and subsequently (e.g. line 60), the EMD omits the word personal treating only doctor as relevant. The address provided at lines 6-8 may also have conveyed, again, without having been designed so to do, information about the wealth of the patient. Although there is no such evidence in this call, there are anecdotal reports that dispatchers untrained in, or non-compliant with, with the MPDS may orient differently to callers with addresses known to be in run-down vs. wealthy areas of cities (Clawson \& Dernocoeur 2003). 
departing from protocol) is designed to include a variant of the category label used by the caller, a doctor (line 60). In so doing he is referring to an unknown person using a standard method for so doing (use of a category) but also thereby providing an implicit account for abandoning the CPR script. This category label is part of and contributes to the explicit account in which the EMD articulates the relevant inferences associated with this category member (he is 'a higher authority' on CPR, line 61) that justify abandoning CPR instruction.

As Schegloff (1996: 449) states, 'different forms of person reference can embody practices for implementing a range of different other activities', that is, actions other than simply referring. We have seen that the caller's use of the categorical reference formulation a personal doctor (line 52) contributes to the action of (polite) resistance to and blocking of the EMD's attempts to provide CPR instruction. We have shown that the EMD understands it this way and subsequently uses the categorical reference $a$ doctor to contribute to his account for abandoning the CPR script. In this instance, then, the use of a categorical person reference fundamentally alters the course of an interaction.

\section{Conclusion}

As Schegloff has said, categories are not mere taxonomic labels: 'they are big-time players in how common-sense culture operates' (Schegloff 2007a: 471), 'the store house and the filing system for the common-sense knowledge that ordinary people ... have about what people are like, how they behave, etc.' (Schegloff 2007a: 469). In the call analysed here the interlocutors' shared orientations to the inferences associated with a member of the category doctor is profoundly consequential for their actions. We have shown that the category doctor is relevantly selected (by the caller) as part of displaying (and accounting for) resistance to an interlocutor's proposed course of action and in order to interrupt that action trajectory. It is so understood by its recipient (the EMD) who immediately halts his course of action and then provides an account for so doing in which he makes explicit an inference associated with the category label. We have shown that (and how) this understanding of common-sense culture embodied in the category doctor is derived from and grounded in analyses of participants' conduct.

Finally, this analysis is the first conversation analytic work on emergency calls to engage with the use of the MPDS protocol, and as such it updates our understanding of contemporary emergency calls and draws attention to the importance of protocols in shaping interaction. We recommend that conversation analysts who hope to contribute to changes in practice and policy in the management of emergency calls should become familiar with this protocol and the medical literature concerning its use. 


\section{Acknowledgements}

IB and SJE independently drew on this call during a master class in conversation analysis taught by $\mathrm{CK}$, the coursework for which required students to locate and analyse a categorical person reference. We are grateful to other members of the class for assistance with the analysis.

\section{Appendix}

Call to 911 Emergency Service made on 25 June 2009

(The caller is designated 'Clr' \& the Emergency Medical Dispatcher as 'EMD')

01 EMD: $\quad$ Fire ' $n$ paramedics thirty three what is the address

02 of your emergency:

03 Clr: $\quad$ Yes Sir. I need to I- I- I need an ambulance

04 as soon as po:ssible sir.

05 EMD: $\quad$ 'kay Sir.=What's your a 1 :ddress.

06 Clr: $\quad$ It's one hundred (.) North Carolwood Drive .hhh

07 Los Angeles California: hh Nine zero zero

08 seven seven.

09 EMD: Did y'say Carolwoodi

$10 \mathrm{Clr}: \quad$ Carolwood Drive yes: hh. $\mathrm{hh}$

$11 \quad(1.5) /(($ Background conversation $))$

12 EMD: $\quad$ Okay Sir ' $n$ what's the phone number you're

13 callin' from.

$14 \quad$ ((deleted material here))

15 EMD: $\quad$ A:nd what's the problem.=Exactly what happened.

$16 \quad(0.5)$

17 Clr: $\quad$ Uh sir I have a: we have a: hh a: a: gentleman

18 here that needs he:lp and he:'s not brea:thing

19 yet. .hhh he's not breathing and we need

20 tuh- we're p- tryin' tah pump him but he's not

$21 \quad(0.2)$ he['s not br ${ }^{\circ}$ eathin'] so: ${ }^{\circ}$

22 EMD:

23 EMD: [O $\mathrm{Oa}: \mathrm{y}$.

Oka:y how old is 'e.

(.)

25 Clr: He's uh fifty years old sir.=

26 EMD: $\quad=$ Fifty: $\quad$ O Oka:y. .hhh (.) He's unconscious he's not brea:thing;

29 Clr:

30 EMD:

Yes he's not breathing sir.

Okay and 'e's not conscious eithe:ri

[(He's) not breathin']

[ $\mathrm{N}$ o h e 's not ] conscious si[:r.]

( ) .hh (1.0)/(Get 'im on the floor)

$$
\text { [O]ka:y. }
$$

Alright. $>$ D'you have 'im- What- Is 'e on the

He's on the bed sir. He's on the [be $: d$.

get ' $\mathrm{im}$ on the floo:r,

[Oka:]y let's

41 Clr: Okay?

42 EMD: $\quad$ Okay let's get 'im down to the floo:r.=I'm gunna 
62

$63 \mathrm{Clr}$ :

64 EMD:

$65 \mathrm{Clr}$ :

66 EMD:

67

$68 \mathrm{Clr}$ :

69

70 Clr:

71 EMD:

$72 \mathrm{Clr}$ :

73 Doc:

74 Clr:

75

76 EMD:

77

78

79 Clr:

80 EMD:

$81 \mathrm{Clr}$ :

82

83 EMD:

84

85 Clr:

86 EMD:

$87 \mathrm{Clr}$ :

88 EMD:

89 Clr:

((recording ends)) help you with C P R right no:w oka::yi

(.)

W::e need 'im to get- we need a: [(

)]

[Yes: We'r] already

on our way the:re. We're on our way.=I'm

gunna do [what I ]can tuh help you over the pho:ne.

[( $)$ ]

We're already on our wa:y. .hhhh Did anybody see hi:mi

(0.2)

Ye:s uhm we have a personal: doctor here with him sir.

[.hhh (But-)]

[Oh y'have a] doctor the:re;

(.)

Yes. But he's not responding to anything. To no: hh no:

He's not responding to: C P R or anythi[ng.]

[Qh:]::: Oka:y.

Well we're on our way the:re. If your guys're doing

C P R and you're (.) instructed by a doctor he has a higher authority than me:.

(.)

[( ) $]$

[And he's] there on sce्ene. [Uh:m ] (0.5) w- was-=

[Okay.]

Did anybody witness what happened $i$

(0.5)

Uh no. Just the doctor sir.

(.)

The doctor's been the only one here.

Okay. So'd the: doctor see what happenedi

Uh uhm Doctor did you see what happened sir.

((from distance)) ( ) co:me.

( ) Sir you just- .hh uh uhm if you can plea:se

uh [uhm]

[We-] We're on our way. We're on our way. I'm jus:t I'm just passin' these questions on to my: pparamedics while they're on the way the:re sir.

Thank you sir. [He- he's p] umpin' he's pumpin' his= [ $\left(\begin{array}{ll}( & )\end{array}\right]$

$=$ che:st but he's not respondin' to anything sir.

Plea:se (.)

Okay. Okay. We're on our way. We're- we're- we're we're lesss than a mile awa:y ' $n$ we'll be there shortly. Thank you sir. Thank [you.]

[Oka ]y sir. [Call us back if you

need any help. ( )

$$
\text { [.hhhh( ) }
$$




\section{References}

Berdowski, Jocelyn, F. Beekhuis, A.H. Zwinderman, J. Tijssen, and R.W. Koster (2009) Importance of the first link: Description and recognition of an out-of-hospital cardiac arrest in an emergency call. Circulation 119: 2096-2102.

Bushnell, Cade (2014) On developing a systematic methodology for analysing categories in talk-ininteraction: Sequential categorization analysis. Pragmatics 24.4: 735-756.

Cheung, Spencer, Charles D. Deakin, Ruby Hsu, Graham W. Petley, and Frank Clewlow (2007) A prospective manikin-based observational study of telephone-directed cardiopulmonary resuscitation. Resuscitation 72: 425-435.

Clawson, Jeff (1986) The hysteria threshold: Gaining control of the emergency caller. Journal of Emergency Medical Services 11: 40.

Clawson, Jeff (1991) Please - don’t ask permission! Journal of the National Academy of EMD, 2.

Clawson, Jeff J. (2000, August 22) Method and system for the entry protocol of an emergency medical dispatch system. United States Patent number 6,106,459.

Clawson, Jeff J., and Kate Boyd Dernocoeur (2003) Principles of Emergency Medical Dispatch. Salt Lake City, UT: Priority Press.

Clayman, Steven E. (2013) Conversation analysis in the news interview. In J. Sidnell, and T. Stivers (eds.), The Handbook of Conversation Analysis. London: Wiley-Blackwell.

Clegg, Gareth R., Richard M. Lyon, Scott James, Holly P. Branigan, and Gerry J. Egan (2014) Dispatchassisted CPR: What are the hold-ups during calls to emergency service dispatchers? Resuscitation 85: 4952.

Deakin, Charles D., Spencer Cheung, Graham W. Petley, and Frank Clewlow (2007) Assessment of the quality of cardiopulmonary resuscitation following modification of a standard telephone-directed protocol. Resuscitation 72: 436-443.

Deutsch, Linda (2011, Nov. 29) Murray gets 4-year sentence, tongue-lashing from judge: Pastor says Michael Jackson's doctor sold out his profession. Today News. Retrieved from http://today.msnbc.msn.com/id/45468611/ns/today-entertainment/\#.UDDckaOaKSo

Eckstein, Marc, Samuel J. Stratton, and Linda S. Chan (2005) Cardiac arrest resuscitation evaluation in Los Angeles: CARE-LA. Annals of Emergency Medicine 45: 504-509.

Garcia, Angela Cora (2015) 'Something really weird has happened': Losing the 'big picture' in emergency service calls. Journal of Pragmatics 84: 102-120.

Graham, Caroline, and Daniel Boffey (2009) Jackson family fallout: Exclusive interview with manager reveals rift over funeral. Retrieved February 22, 2010 from http://www.dailymail.co.uk/news/worldnews/article-1197571/Jackson-family-fallout-Exclusiveinterview-manager-reveals-rift-funeral.html\#ixzz0gIVesRXK

Hester, Stephen, and Peter Eglin (1997) Membership categorization analysis: An introduction. In S. Hester, and P. Eglin (eds.), Culture in Action: Studies in Membership Categorization Analysis. Washington, DC: University Press of America, pp. 1-24.

Heward, A, M. Damiani, and C. Hartley-Sharpe (2004) Does the use of the Advanced Medical Priority Dispatch System affect cardiac arrest detection? Emergency Medicine Journal 21.1: 115-118. 
Kitzinger, Celia (2005) Heteronormativity in action: Reproducing the heterosexual nuclear family in after-hours medical calls. Social Problems 52: 477-498.

Kitzinger, Celia, and Rose Rickford (2007) Becoming a "bloke": The construction of gender in interaction'. Feminism and Psychology 17: 214-223.

Kitzinger, Celia (2008) Developing feminist conversation analysis: A response to Wowk. Human Studies 31.2: 179-208.

Lerner, E. Brooke, Michael R. Sayre, Jane H. Brice, Lynn J. White, Amy J. Santin, Anthony J. Billitier IV, and Samuel D. Cloud (2008) Cardiac arrest patients rarely receive chest compressions before ambulance arrival despite the availability of pre-arrival CPR instructions. Resuscitation 77: 51-59.

Lerner, Gene H., and Celia Kitzinger (2007) (eds.) Referring to Self and Others in conversation: Special Issue. Discourse Studies 9.

Moore, Solomon (2009, August 28) Jackson's death ruled a homicide. The New York Times. Retrieved from: http://www.nytimes.com/2009/08/29/us/29jackson.html

Paoletti, Isabella (2012) Operators managing callers' sense of urgency in calls to the medical emergency number. Pragmatics 22: 671-695.

Raymond, Chase Wesley (2014) Negotiating entitlement to language: Calling 911 without English. Language in Society 43: 33-59.

Roppolo, Lynn P., Paul E. Pepe, Nicole Cimon, Marc Gay, Brett Patterson, Arthur Yancey, and Jeff J. Clawson (2005) Modified cardiopulmonary resuscitation (CPR) instruction protocols for emergency medical dispatchers: Rationale and recommendations. Resuscitation 65: 203-210.

Sacks, Harvey (1972a) An initial investigation of the usability of conversational data for doing sociology. In D.N. Sudnow (ed.), Studies in Social Interaction. New York: Free Press, pp. 31-74.

Sacks, Harvey (1972b). On the analyzability of stories by children. In J. Gumperz, and D. Hymes (eds.), Directions in Sociolinguistics: The Ethnography of Communication. New York: Holt, Rinehart and Winston, pp. 325-345.

Sacks, Harvey (1995) Lectures on Conversation. Oxford: Blackwell.

Sacks, Harvey, and E.A. Schegloff (1979) Two preferences in the organization of reference to persons in conversation and their interaction. In G. Psathas (ed.), Everyday Language: Studies in Ethnomethodology. New York: Irvington, pp. 15-21.

Schegloff, Emanuel A. (1987) Analyzing single episodes of interaction: An exercise in conversation analysis. Social Psychology Quarterly 50: 101-114.

Schegloff, Emanuel A. (1996) Some practices for referring to persons in talk-in-interaction: A partial sketch of a systematic. In B.A. Fox (ed.), Studies in Anaphora. Amsterdam: John Benjamins Publishing Company, pp. 437-485.

Schegloff, Emanuel A. (2007a) A tutorial on membership categorization. Journal of Pragmatics 39: 462482.

Schegloff, Emanuel A. (2007b) Categories in action: Person reference and membership categorisation. Discourse Studies 9: 433-461.

Schegloff, Emanuel A. (2007c) Sequence Organisation in Interaction: A Primer in Conversation Analysis I. Cambridge: Cambridge University Press. 
Silberman, Seth Clark (2007) Presenting Michael Jackson. Social Semiotics 17: 417-440.

Sokol, Daniel K. (2009) Hippocrates, Michael Jackson, and medical ethics. British Medical Journal 339: 541.

Stivers, Tanya (2007) Alternative recognitionals in person reference. In N.J. Enfield, and T. Stivers (eds.), Person Reference in Interaction: Linguistic, Cultural, and Social Perspectives. Cambridge: Cambridge University Press, pp. 73-96.

Stokoe, Elizabeth (2009) Doing actions with identity categories: Complaints and denials in neighbor disputes. Text and Talk 29: 75-97.

Stokoe, Elizabeth (2012) Moving forward with Membership Categorization Analysis: Methods for systematic analysis. Discourse Studies 14.3: 277-303.

Telegraph, The (2011, September 29) Conrad Murray on trial for involuntary manslaughter of Michael Jackson: Sept 29 as it happened. Retrieved from:

http://www.telegraph.co.uk/culture/music/michael-jackson/8869552/Conrad-Murray-on-trial-forinvoluntary-manslaughter-of-Michael-Jackson-Sept-29-as-it-happened.html

Tracy, Karen, and Sarah J. Tracy (1998) Rudeness at 911: Reconceptualizing face and face attack. Human Communication Research 25: 225-251.

Watson, Richard E., Maureen McNeil, and Carol Biancalana (2003) Emergency Medical Services Dispatch Program Guidelines. Sacramento CA: California EMS Authority. Retrieved from: http://www.emsa.ca.gov/media/default/pdf/emsa132.pdf

Whalen, Marilyn R., and Don H. Zimmerman (1990) Describing trouble: Practical epistemology in citizen calls to the police. Language in Society 19: 465-492.

Whalen, Marilyn R., and Don H. Zimmerman (1987) Sequential and institutional contexts in calls for help. Social Psychology Quarterly 50: 172-185.

Whalen, Marilyn R. (1988) When words fail: A single case analysis. Social Problems 35: 335-362.

Wilkinson, Sue, and Celia Kitzinger (2003) Constructing identities: A feminist conversation analytic approach to positioning in action. In R. Harré, and F. Moghaddam (eds.), The Self and Others. Westpoint, CT: Praeger, pp. 157-180.

Zimmerman, Don H. (1984) Talk and its occasion: The case of calling the police. In Deborah Schiffrin (ed.), Meaning, form and use in context: Linguistic applications. Georgetown University Round table on languages and linguistics 35. Washington, DC: Georgetown University Press, pp. 210-28.

Zimmerman, Don H. (1992) The interactional organization of calls for emergency assistance. In P. Drew, and J. Heritage (eds.), Talk at Work. Cambridge: Cambridge University Press, pp. 418-469.

ISRAEL BERGER is a Lecturer in Qualitative Health Research and final year medical student at the University of Sydney (Australia) and completed his Ph.D. in psychology at the University of Roehampton (UK). His research involves health communication conducted through a variety of media, including public health interventions, patient-provider communication, and counselling/psychotherapy; conversation analytic methodology; and research and medical ethics, particularly in the areas of autonomy and consent. 
Address: VELiM, Medical Foundation Building, University of Sydney, Camperdown NSW 2006, Australia. E-mail: israel@israelberger.com

SONJA J. ELLIS is working at the Australian Institute of Professional Counsellors and has published a number of journal articles mainly in the field of lesbian and gay psychology. Her work is featured in three of the leading texts in this field, and she is also co-author of the first undergraduate textbook on LGBTQ psychology. She is currently undertaking research which explores social interaction in the context of telephone calls to emergency services, and using conversation analysis.

Address: Locked Bag 15, Fortitude Valley, Qld 4006, Australia. E-mail: sonja@aipc.net.au

CELIA KITZINGER is Professor of Sociology at the University of York (UK) and has held visiting appointments at the universities of British Columbia (Canada) and Auckland (New Zealand). She also regularly lectures and teaches graduate and researcher training workshops and short courses and summer schools in the UK and internationally, including advanced courses in conversation analysis at the University of York for external students. Her most recent research explores family decision-making about patients with traumatic brain injuries.

Address: University of York, Sociology, Heslington, York YO10 5DD, United Kingdom. E-mail: celia.kitzinger@york.ac.uk 